\title{
Interest of a Rheokinetic Study for the Development of Thermoplastic Composites by T-RTM
}

\author{
Matthieu Thomassey ${ }^{1, *}$, Baptiste Paul Revol ${ }^{1,2}$, Frédéric Ruch $^{1}$, Julia Schell $^{3}$, Michel Bouquey ${ }^{4}$ \\ ${ }^{1}$ Engineering of Polymers and Composites, France \\ ${ }^{2}$ Materials Science Institute of Mulhouse, UMR-CNRS 7361, France \\ ${ }^{3}$ Engineering of Polymers and Composites (Techno Campus Composite), Cetim, France \\ ${ }^{4}$ Macromolecular Chemistry Accuracy, Institute Charles Sadron, France
}

Copyright $\bigcirc 2017$ by authors, all rights reserved. Authors agree that this article remains permanently open access under the terms of the Creative Commons Attribution License 4.0 International License

\begin{abstract}
Until today, thermosetting polymer based composites were predominant, but are faced with environmental rules more stringent (COV, recyclability...). Thermoplastic composites are a good answer to the evolution of environmental rules, and have an increased need due to their improved properties compared to thermosets. One thermoplastic process route offered is the injection of monomers with a low viscosity (around 10mPa.s): Thermoplastic-Resin Transfer Molding (T-RTM). Currently, reactive thermoplastics are not technically matured for industrial applications. Indeed, their chemistry and rheology especially in the presence of fibers are not well studied and understood. The reaction time is the most crucial parameter for a well-controlled injection and the viscosity should stay low until full impregnation is achieved. The other point is the reaction kinetics. The faster the reaction, the more reaction heat is released in less time which results in overheating in the center of very thick parts. In a first approach the pure polymer is investigated. To better understand the reaction time during an injection process, rheological measurements were achieved at different shear rates and temperatures. In parallel, the reaction kinetics through the exothermic reaction during polymerization are modelled and experimentally verified.
\end{abstract}

Keywords Polyamide, Resin Transfer Molding, Rheology, Thermoplastic Composites, Modeling

\section{Introduction}

Until now, thermosetting polymer based composites were predominant, but are faced with environmental regulations, REACH restrictions on solvents, recycling, waste reduction... All these drawbacks promote the use and the developments of composite materials based on thermoplastic which are polymers with a higher recycling capacity. Moreover, thermoplastic composite materials can be implemented with reduced cycle times with different technologies in cleaner processes. Recent advances in the development and processing techniques of thermoplastic composites have increased their use in structural and semi-structural applications, the traditional domain of metal alloy or thermoset composites [1].

Although many works have been done on these subjects [1-5], the RTM technology based reactive thermoplastic resins is still not enough mature to lead to industrial applications. A major difficulty in processing thermoplastic composites is to achieve cost-effective high quality impregnation of the fiber reinforcement by the matrix resin [6].

In the case of thermoplastic resins, it is well known that the polymer resins are much more viscous than reactive thermosetting resins. To circumvent this difficulty, two strategies are generally adopted: either to reduce the viscosity of certain polymer resins up to $10 \mathrm{mPa}$.s, and/or to control the reactive thermoplastic monomers in RTM process. This difficulty increases if fiber preforms are placed in the mold with high volume rate (up to $60 \%$ vol.) to manufacture thermoplastic composites.

Moreover, it is well known that the anionic polymerization process starts during the mold filling phase, leading to a sharp increase in viscosity of the reaction mixture and limiting the capacity of filling and also fibers impregnation with the thermoplastic reactive mixture. Currently, for reinforced thermoplastic composites, the RTM injection involves two steps:

1. Progressive filling of the mold with the reactive mixture containing fiber reinforcements,

2. Polymerization of the reaction mixture in contact with the reinforcement.

From a technical point of view, the challenge could be to imagine processes in which the phase of "injection/fibers impregnation" of the reaction mixture is delayed or dissociated to the "polymerization" phase in the best case. 
The goal of this approach is, on the one hand, to improve the fibers impregnation in the case of reinforced composites and, on the other hand, limiting the material shrinkage effect which causes defects or weaknesses in the materials.

In this work, a theoretical and experimental approach T-RTM injection has been done to determine all needed parameters for modeling the injection process. With the help of a numerical tool, it is intended to find a strategy to optimize and delay the polymerization reaction time during the T-RTM injection. This strategy aims to maintain the viscosity as low as possible for the reaction mixture during the injection into the mold. This strategy has to improve the residence time and thus the impregnation time of the fibers by the reactive mixture before the polymerization is too advanced and consequently locks the impregnation. In an idealistic case, this new view of the T-RTM injection strategy could be decomposed into three steps:

1. Filling of the mold containing reinforcements (or not) with the reaction mixture,

2. Reinforcements impregnation by the reaction mixture with lowest viscosity as possible,

3. Bulk polymerization of the reaction mixture.

This strategy is intended to dissociate the filling phase of the mold and the reinforcement's impregnation phase from the polymerization phase by controlling the delay of the progression of the reaction polymerization. Understanding and controlling reactive T-RTM requires a thorough study of the thermal and rheological behaviors of the pure resin kinetic as a function of time and temperature before introducing fibers inside the matrix. A better understanding of the different phenomena could be obtained through kinetic information. Indeed, thermal and viscosity rise are determined by the polymerization kinetic. This is linked to the chemical reaction advancement.

The scientific challenge in this work is to devise a new concept for the implementation of a reactive resin able to postpone the polymerization reaction in a hot mold adapted to the production of unreinforced thermoplastic parts/reinforced thermoplastic composites. This way is a complete scientific break up with the current proposed solutions, which are consisting into play on the slopes of the imposed mold temperature according to the thickness of the part [7]. In classical injection process, this temperature strategy leads quickly to an inability to produce massive technical parts because the cycle time which grows exponentially with the thickness of the mass to be polymerized. Indeed, forming massive technical parts induces temperature gradients in the mold during polymerization, due to the low thermal conductive nature of plastic materials. In this case, the new strategy developed in this work sees its utility in the filling of parts with complex shapes and geometries.

This work is especially focused on the anionic polymerization by ring opening of a lactam from commercial products, i.e. AP-Nylon ${ }^{\mathbb{B}}$ Caprolactam. All needed products are from Brüggemann Chemicals. The goal of this work is to introduce a possible approach of polymerization delayed pure AP-Nylon ${ }^{\circledR}$ system in the case of an implementation by T-RTM. Moreover, the extreme fluidity of this reactive mixture, the low pressure and temperature used in the T-RTM process and the delayed polymerization method developed in this work have all advantages to lead to the emergence of materials-process solutions for the manufacture of technical parts, small, massive, with a complex shape and even functionalizable. This solution is also being compatible with the presence of fibers to be extended to composite materials. This will meet the requirements of small production rates, and limited cost control, recyclability.

\section{Experimental Parts}

\subsection{Materials}

The reactive mixture consists of three components: $\varepsilon$-caprolactam (CL) named AP-Nylon ${ }^{\circledR}$ Caprolactam solid $\left(99 \%, \mathrm{Mw}=113.16 \mathrm{~g} \cdot \mathrm{mol}^{-1}\right)$ as monomer, Brüggolen $\mathrm{C} 10$ (sodium caprolactamate, $1 \mathrm{~mol} \cdot \mathrm{kg}^{-1}$ concentration in caprolactam) as initiator and Brüggolen $\mathrm{C} 20 \mathrm{P}$ (N, N-hexane-1, 6-diylbis

(hexahy-dro-2-oxo-1H-azepine-1-carboxamide), 2mol. $\mathrm{kg}^{-1}$ concentration in caprolactam) as activator. Brüggemann Chemical (Germany) supplied all products in sealed polyethylene-lined aluminum drums. After opening, the CL, $\mathrm{C} 10$ and $\mathrm{C} 20 \mathrm{P}$ sealed boxes are properly closed and stored in freezing bag filled with dry argon atmosphere. All products are used as received without further processing or purification. For all measurements, the same sample preparation of the reactive mixture is used. With respect to caprolactam, 4.5 weight \% Brüggolen $\mathrm{C} 10$ is added as activator, together with 3.0 weight \% Brüggolen C20P.

\subsection{Sample Preparation for Rheological Measurements}

All raw products are placed in a commercial blender and mixed together during $t=5 \mathrm{~s}$. By this way, a fine white powder is obtained. A homogenous distribution of activators and initiators in the CL with a narrow granulometry distribution is assumed. The reactive mixtures were fresh daily prepared. Samples are immediately sealed in freezing bag filled with dry argon to limit oxygen contamination.

\subsection{Sample Preparation with Mini-Mixing Unit (MMU) for Semi-Adiabatic Polymerization and Instrumented Mold}

In order to prepare the reactive mixture in a semi-industrial way, a lab-scale mixing unit, Mini Mixing Unit (MMU), provided by Bronk Industrial BV (The Netherlands) was used. Two liquid mixtures, $\mathrm{CL} / \mathrm{C} 10$ and $\mathrm{CL} / \mathrm{C} 20 \mathrm{P}$, under a nitrogen atmosphere at $\mathrm{T}=110^{\circ} \mathrm{C}$ were placed in two distinct tanks. Liquid mixtures are under a 
permanent stirring in each tank with its own mixing mechanism. Before the mixing procedure, the stirring is stopped and both tanks are individually degassing. To ensure a ratio 1:1, the injection pistons have the same displacement speed. The two materials feeds are mixed by using a long static mixer. The liquid reactive mixture at the exit of the static mixer is directly either injected into the thermal reactor or into the instrumented mold (see next paragraph).

\subsection{Experimental Setups}

\subsubsection{Rheological Measurements}

All rheological experiments are performed with a strain-controlled rheometer (ARES, Rheometric Scientific). Disposable aluminum cone-plate geometries have been specifically designed for measuring the polymerization kinetics and to avoid evaporation of the reagent mixture. A specific Newtonian oil bath is used as isolator in a double wall on the lower part. Conical measuring geometries have a diameter of $25 \mathrm{~mm}$. All elements are preheated to $\mathrm{T}=90^{\circ} \mathrm{C}$ and zero gaps are adjusted before all measurements. After these steps, reactive powder is introduced as fast as possible inside the measuring geometry. A waiting time of $t=15 \mathrm{~s}$ is respected to ensure that the mixture is in a liquid state to set the gap easier. Rotational measurements are realized. Shear viscosity growth during anionic polymerization of caprolactam is measured under isothermal conditions at different polymerization temperatures. The following tests were conducted: (i) as first step a pre-shear rotational test in controlled shear rate mode is realized with the following parameters: $\dot{\gamma}=10 \mathrm{~s}^{-1}, t=300 \mathrm{~s}, \mathrm{~T}=90^{\circ} \mathrm{C}$. This pre-shear is directly followed by (ii) measuring rotational test in controlled shear rate mode with the parameters: $\dot{\gamma}=0.1 / 1 / 10 / 100 \mathrm{~s}^{-1}, t=600 \mathrm{~s}, \mathrm{~T}=130 / 140 / 150 / 160 / 170^{\circ} \mathrm{C}$. In each case, measurements were automatically terminated when the instrument sensed overload on the torque load cell.

\subsubsection{Semi-Adiabatic Polymerization}

In order to mimic the injection phase in the process, semi-adiabatic polymerization tests were performed to investigate the semi-adiabatic temperature built-up. As described in [8], the test setup for the semi-adiabatic polymerization was adapted to an injection with the MMU in a thick stainless steel reactor (diameter $=30 \mathrm{~mm}$, height $=250 \mathrm{~mm})$. A reactor lid was especially designed to ensure the airtightness of the system during polymerization and the correct placement of the type $\mathrm{K}$ insulated thermocouple in the middle of the reactor. The polymerization mixture was prepared in the mini-mixing unit with the conditions described above. To simulate the polymerization reaction during a process injection, the reactor filled with the reactive mixture was placed in an oil bath set to $\mathrm{T}=140 / 150 / 160 / 170^{\circ} \mathrm{C}$ (temperatures corresponding to mold temperature). The temperature of the mixture was recorded in situ every second with National Instruments LabVIEW Signal Express 2012 data acquisition device.

\subsubsection{T-RTM Processing in an Instrumented Mold}

To compare semi-adiabatic temperature built-up and validate all simulation data, an instrumented mold was designed to measure in situ the temperature increase due to exothermic heat produced by the polymerization at different places at the surface and in the bulk. A useful surface of the mold was set to $400 \times 260 \mathrm{~mm}$ and $4 \mathrm{~mm}$ thick. The temperature sensors are type $\mathrm{K}$ insulated thermocouples from Kistler Instrument. They are placed in the mold's axe. Different locations could also be chosen to enable a scan of the entire mold and to follow-up the temperature in all points and at all times. The polymerization mixture was prepared in the mini-mixing unit with the conditions described above. To obtain a homogenous temperature, the mold was placed in a hot press which was kept at the same temperature as the semi-adiabatic polymerization in thermal reactor. To ensure a good polymerization, the mold was kept inside the hot press during $t=15 \mathrm{~min}$. The temperature of the mixture was recorded in situ every second with Agilent data acquisition device.

\section{Experimental Characterizations}

\subsection{Degree of Conversion}

To determine the degree of conversion $(\beta)$ of APA6 polymerized in each sample, a chemical approach was chosen. Samples are weighted $\left(\mathrm{m}_{t o t}\right)$ just before being dissolved in formic acid during $t=120 \mathrm{~min}$ at $\mathrm{T}=40^{\circ} \mathrm{C}$. After that, under magnetic agitation, solution is precipitated in ethanol. After filtering the solution of ethanol, the obtained residue is weighted $\left(\mathrm{m}_{\text {res }}\right)$. The degree of conversion is calculated as the ratio of $\mathrm{m}_{\text {res }} / \mathrm{m}_{\text {tot }}$. This method dissolves completely PA6 and accesses the monomer trapped in the bulk of the material.

\subsection{Degree of Crystallinity}

A Mettler Toledo TC15 Controller differential scanning calorimetry (DSC) was used to characterize the thermal behavior and especially the degree of crystallinity $\left(\mathrm{X}_{\mathrm{c}}\right)$. The samples were heated from $\mathrm{T}=40^{\circ}$ to $\mathrm{T}=250^{\circ} \mathrm{C}$ at $10^{\circ} \mathrm{C} \cdot \mathrm{min}^{-1}$. The device is temperature and heat flow calibrated using indium and zinc. Aluminum standard crucibles $40 \mu \mathrm{L}$ provided by Mettler Toledo are used in all experiments. Sample masses are between $\mathrm{m}=10$ and $20 \mathrm{mg}$. For each measurement, filled crucibles are weighed before and after in order to control that there is no loss of material. The degree of crystallinity $\left(\mathrm{X}_{\mathrm{c}}\right)$ was calculated by comparing the experimental heat of fusion $(\Delta \mathrm{H})$ to the theoretical value for a $100 \%$ crystalline sample $\left(\Delta \mathrm{H}_{100}\right)$. The experimental heat of fusion was measured by integrating the area under the peaks of DSC thermogrammes. The melting enthalpy of fully crystalline PA6 was chosen equal to $\Delta \mathrm{H}_{100}=190 \mathrm{~J} / \mathrm{g}$ [9]. 


\section{Modeling}

The aim of the modeling is to predict the temperature increase due to polymerization and crystallization. High temperatures which can easily arise in the center of a very thick part can overheat and damage the part. Thermal gradients are to avoid too, as they induce thermal stress which can deform the part. In the simulation only the heat transfer during heating and curing is taken into account. The injection and filling are not considered as they are too short to influence the thermal history.

Numerical simulations of the heat transfer process including the heat sources of the polymerization and crystallization are performed using the FE-program COMSOL. Assuming that polymer flow is negligible, there is no convection, and that the polymer and the fibers are at the same temperature, the energy balance equation is reduced to the equation of transient heat transfer:

$$
\rho\left(C_{p}\right) \frac{\partial T}{\partial t}=\nabla(k . \nabla T)+Q
$$

with $\rho$ the density, $C_{p}$ the specific heat, $k$ the thermal conductivity, $T$ the temperature and $Q$ a source term. In the studied case, $Q$ corresponds to the internal heat generated by the polymerization and the crystallization:

$$
Q=\rho \cdot\left(H_{\text {poly }} \cdot \frac{\partial \beta}{\partial t}+H_{\text {crist }} \cdot \frac{\partial \alpha}{\partial t} \cdot \beta_{\text {real }}\right)
$$

with $H_{\text {poly }}$ the enthalpy of the polymerization and $H_{\text {crist }}$ the one of the crystallization. To calculate the generated heat $Q$, the corresponding $H$ is multiplied with the corresponding changing rate of the generated phase. These changing rates are determined by the kinetics of the phase change. The autocatalytic polymerization is described by the model of Kamal-Sourour:

$\frac{\partial \beta}{\partial t}=\left(A_{1} \cdot \exp \left(-\frac{E_{1}}{R T}\right)+A_{2} \cdot \exp \left(-\frac{E_{2}}{R T}\right) \cdot \beta^{n}\right)\left(\beta_{\max }-\beta\right)^{n}$

with $A_{1}$ and $A_{2}$ the pre-exponential factors, $E_{1}$ and $E_{2}$ the activation energies, $m$ and $n$ the kinetic exponents of the reaction describing the order of the reaction. Taking into account that the CL polymerizes at temperatures above $\mathrm{T}=140^{\circ} \mathrm{C}$, the polymerization and its heat generation are considered at $\mathrm{T}>140^{\circ} \mathrm{C}$. For the non-isothermal crystallization behavior the model of Lee and Kim is taken [10]:

$$
\frac{\partial \alpha}{\partial t}=A_{c r i} \cdot \exp \left(-\frac{E_{D}}{R T}\right) \cdot \exp \left(-\frac{\Psi \mathrm{T}_{m}^{0}}{T\left(T_{m}^{0}-T\right)}\right) \cdot \alpha^{2 / 3} \cdot\left(\alpha_{e q}-\alpha\right)
$$

with $\Psi$ a crystallization constant, $T_{m}^{0}$ the temperature of maximum rate of crystallization, $T_{k}$ the melting temperature of the reaction mixture, $E_{D}$ the activation energy for chain diffusion, $\alpha_{e q}$ the equilibrium crystallinity and $\beta_{\mathrm{ci}}$ is the conversion at which the crystallization starts and $\alpha_{\max }$ is theoretically equal to 0.46 . The crystallites are built from APA. This effect is taken into account by these equations:

$$
\alpha_{\text {reel }}=\alpha \cdot \beta \text { and } \beta_{\text {reel }}=\beta-\alpha_{\text {reel }}
$$

The resin properties $\left(\rho, k, C_{p}\right)$ are calculated by a mixing law taking into account the amount of caprolactam (CL) and anionic polyamide 6 (APA). For example the equation for the density:

$$
\rho(T, \beta)=\rho_{C L}(T) \cdot(1-\beta)+\rho_{A P A}(T) \cdot \beta
$$

The equations describing the temperature dependency and the values of material properties are taken from [10] and [11].

\section{Results and Discussion}

\subsection{Kinetics Study of the Activated Polymerization of $\varepsilon$-Caprolactam}

The kinetic parameters of the reaction depending on the polymerization temperatures were determined on samples of the mass in the thermal reactor. The objective of these experiments is to obtain the activation energy and constant rate as function of polymerization temperature.

In figure 1 , it can immediately be observed that whatever the bath temperature, there are two steps in the temperature evolution: a phase during which the temperature evolution is almost the same for all bath temperatures, and a phase of very high exothermal peak (over $\Delta \mathrm{T}=50^{\circ} \mathrm{C}$ ) which may eventually be assigned to the polymerization reaction of the reactive mixture. By isoconversional for these curves, an apparent picture of the physicochemical effects during polymerization could be obtained and represented in an Arrhenius diagram (figure 2).

In figure 2, whatever the bath temperature, two distinct zones are observables. For colder temperatures (zone 2), curve slopes are steeper and seem almost identical. In zone 1, the slopes are less pronounced. It is possible to determine the activation energy by a simple linear regression. The results obtained are shown in the figure 3.

As we could observe in figure 3 for the zone 1, for higher bath temperatures, the decrease of the apparent activation energy becomes more significative. The value of the activation energy obtained seems to correspond to the crystallization phenomenon. Indeed, $\mathrm{E}_{a, \text { cryst. }}=16.1 \mathrm{~kJ} \mathrm{~mol}^{-1}$ is found [12]. The same work was done for the curves in zone 2 (red points). It also clear that the values are almost equal to the value of the polymerization activation energy of the, $\mathrm{E}_{a \text {, }}$ polym. $=56.5 \mathrm{~kJ} . \mathrm{mol}^{-1}[13]$.

If it carries out the sum of the values $\mathrm{E}_{a}$ of the apparent activation energies obtained in both zones, the total activation energy calculated is equal to $\mathrm{E}_{a \text {,total }}=68 \mathrm{~kJ} \mathrm{~mol}^{-1}$. This value is close to the value of the reaction activation energy found in the literature for an equivalent system $\mathrm{E}_{\text {a,react. }}=63.5 \mathrm{~kJ} . \mathrm{mol}^{-1}[8]$. This approach is particularly enhanced by the fact that one obtains overall activation energies of the same order of magnitude as the values $\mathrm{E}_{a}$ given in the literature for the same system, and this approach dissociates every phenomenon. 


\subsection{Rheological Study of the Activated Polymerization of $\varepsilon$-Caprolactam}

In a first part of the rheological study, flow measurements were performed at a constant shear rate, $\dot{\gamma}=10 \mathrm{~s}^{-1}$, but different polymerization temperature $\mathrm{T}_{\text {polym }}=$ $140 / 150 / 160 / 170^{\circ} \mathrm{C}$ (figure 4).

In a second part of this work, the influence of the shear rate on the reactive mixture during polymerization was also studied. For this, flow measurements were performed at a constant polymerization temperatures $\left(\mathrm{T}_{\text {polym }}=\right.$ $\left.140 / 150 / 160 / 170^{\circ} \mathrm{C}\right)$ at different shear rates $\dot{\gamma}=0.1 / 1 / 10 / 100$ s- 1 (figure 5).

Immediately, it is apparent that all cases in all cases during the pre-shear stage no variation in viscosity is observed. This means that at this temperature and with the pre-shear rate applied, no polymerization occurs during a period of $t=300 \mathrm{~s}$. In the two studies, beyond $t=0 \mathrm{~s}$, it is found that the change in viscosity can be divided into two distinct parts: an induction phase where there is no viscosity change and a phase in which the viscosity changes considerably with time.

In the case of temperature influence (figure 4), beyond $t=0 \mathrm{~s}$, time corresponding to the beginning of the change in temperature to the polymerization temperature, it is found that the latter influences the change in viscosity over time. It is clearly seen that the curves obtained can be cut into two parts: a first portion where the viscosity does not change when the polymerization has started, and another where the viscosity has a strong divergence. Taking as reference a viscosity of $\eta=10000 \mathrm{~Pa}$.s (arbitrary choice), the observed time interval is $t=100 \mathrm{~s}$ between the viscosity of a system polymerized at $\mathrm{T}=170^{\circ} \mathrm{C}$ and a polymerized system at $\mathrm{T}=140^{\circ} \mathrm{C}$. These curves can be approximated by a simple model of the type $\eta=\eta_{0}$.exp $(k t)$, where $\eta_{0}$ is the zero shear viscosity and $k$ is a constant feature of the evolution of the system worth $17.3 \pm 0.2$ in our case. This value is in agreement with the literature [14], since there is a 19.5 value in equivalent systems.

In the case of shear rate influence (figure 5), it is found that for an isothermal polymerization temperature at $\mathrm{T}=160^{\circ} \mathrm{C}$, like the temperature, the shear rate influences the change in viscosity over time. As previously, if a reference viscosity $\eta=10000 \mathrm{~Pa}$.s (arbitrary choice) is chosen, the time lag observed between the viscosity of a polymerized system under a shear rate at $\dot{\gamma}=100 \mathrm{~s}^{-1}$ and at $\dot{\gamma}=0.1 \mathrm{~s}^{-1}$ is equal to $\Delta t$ $=160 \mathrm{~s}$. It appears that the influence of the shear rate is far from negligible during polymerization, and could be more important than the effect of temperature.

Following the rheological study at different temperatures of polymerization, it was found that the shear rate seemed to strongly influence the kinetics of polymerization. To compare all the results obtained through various tests, it was chosen arbitrarily a viscosity $\eta=100$ Pa.s (reference viscosity taken in ASTM D4473). The summary of results is presented in the following graph:

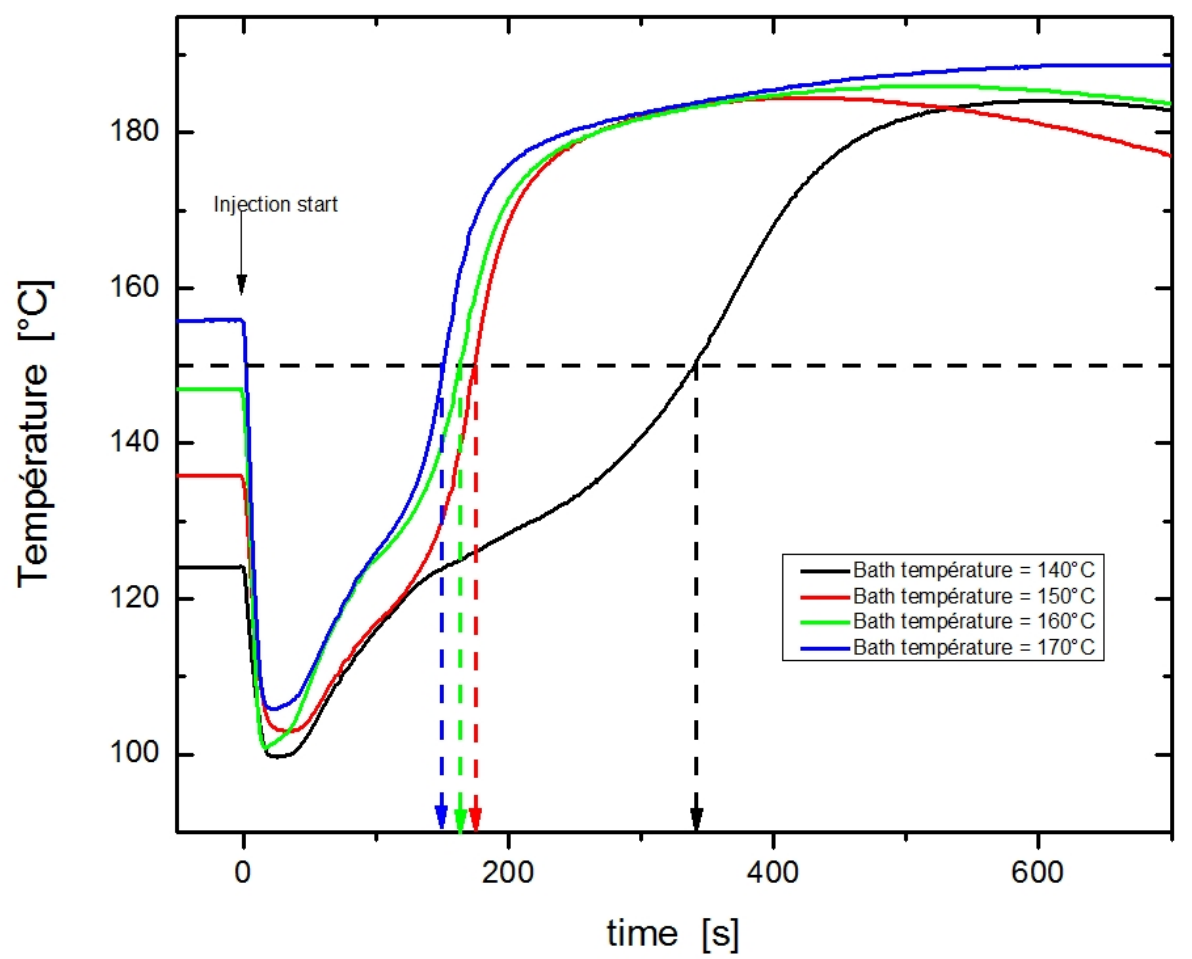

Figure 1. Evolution of the temperature measured by the thermocouple at the center of the thermal reactor as a function of time for four different bath temperatures $\left(\mathrm{T}=140 / 150 / 160 / 170^{\circ} \mathrm{C}\right)$. 


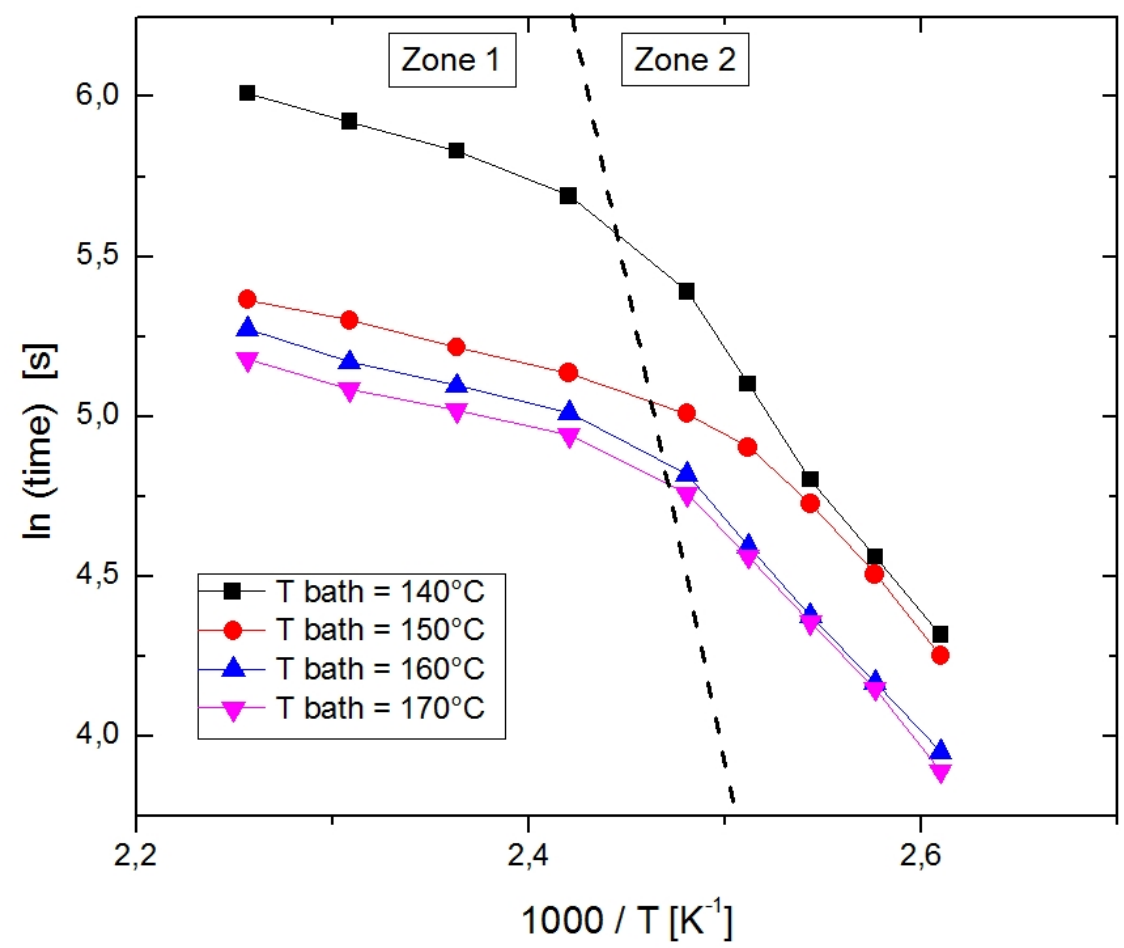

Figure 2. Arrhenius diagram of the apparent polymerization reaction for different bath temperatures

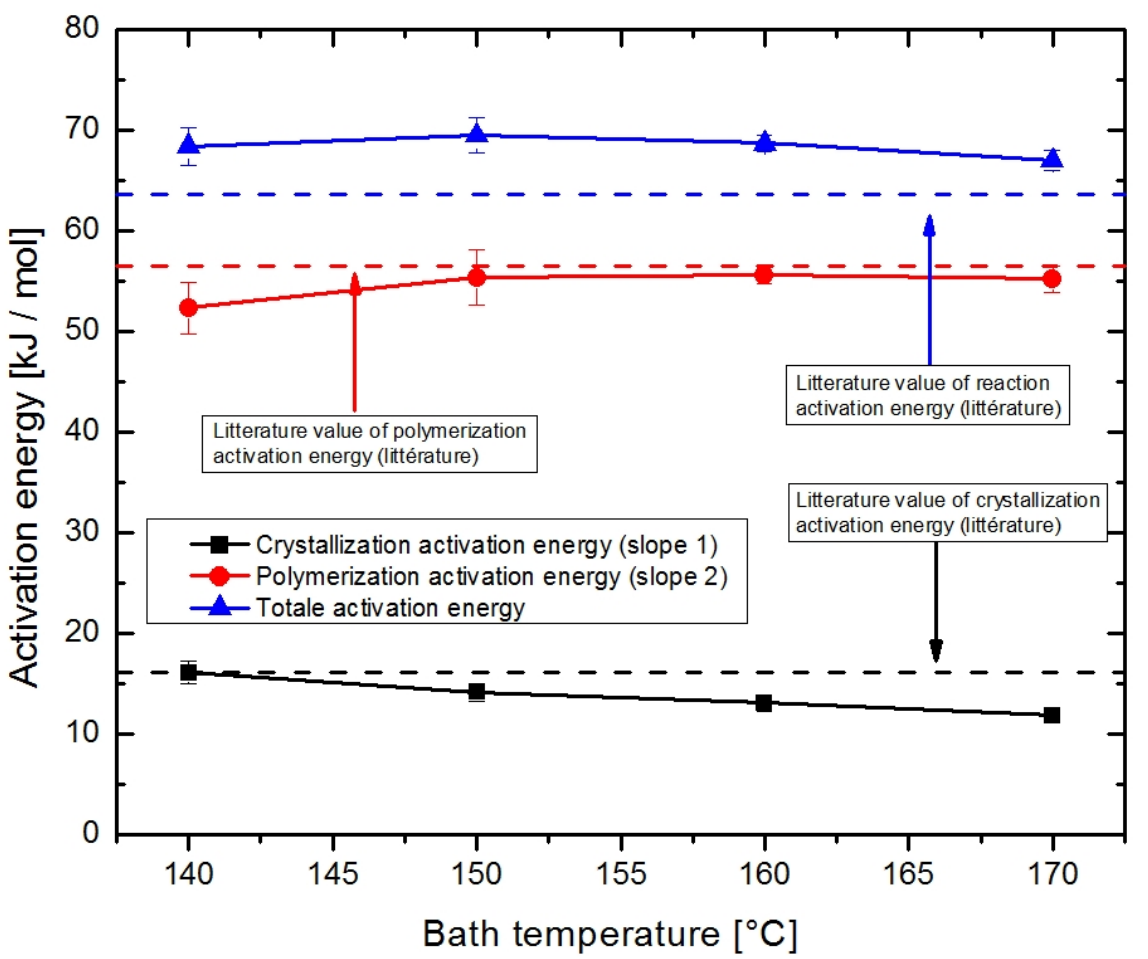

Figure 3. Activation energies of physicochemical processes as function of different bath temperatures compared to the literature values. 


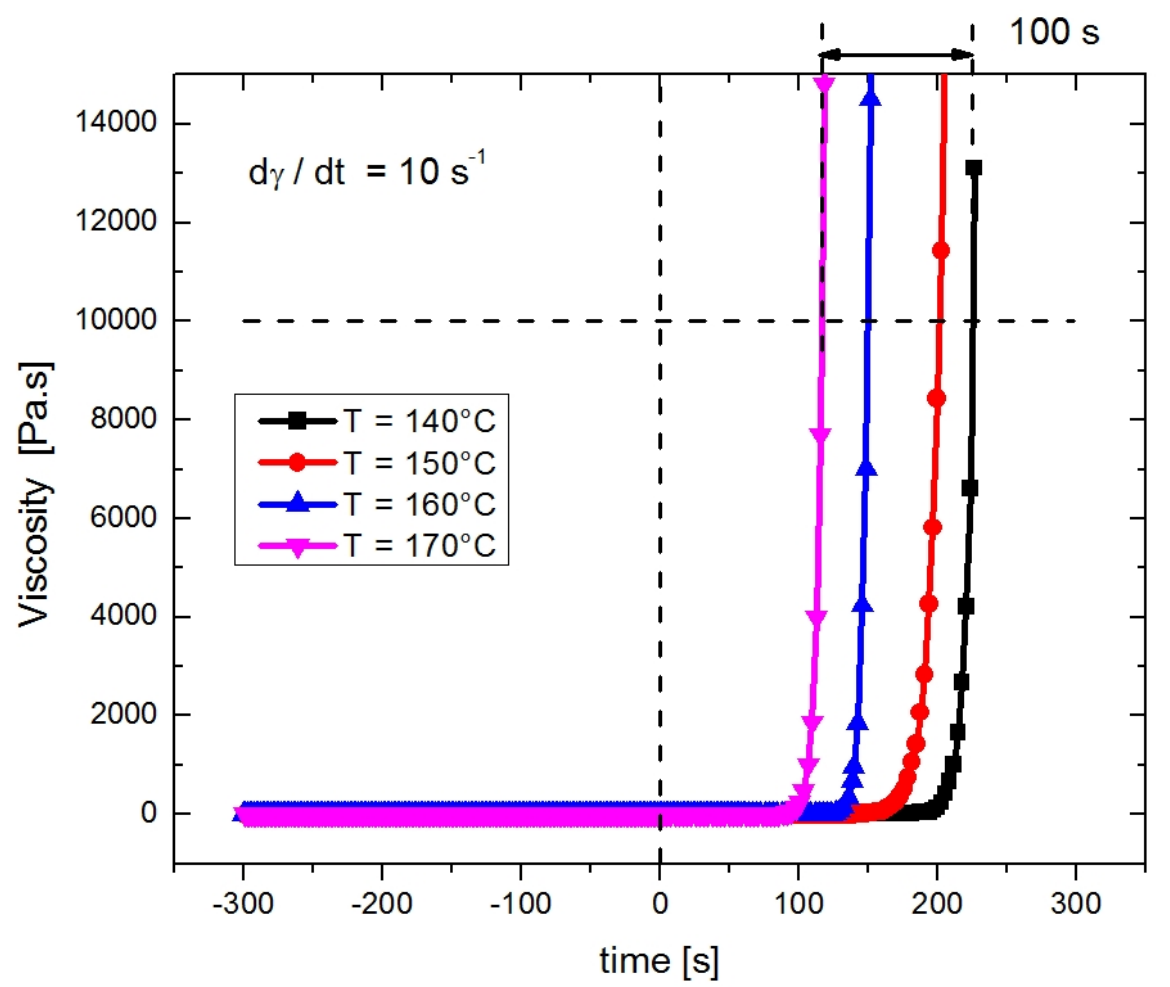

Figure 4. Viscosity evolution at different polymerization temperatures as function of time at a constant shear rate $\dot{\gamma}=10 \mathrm{~s}^{-1}$.

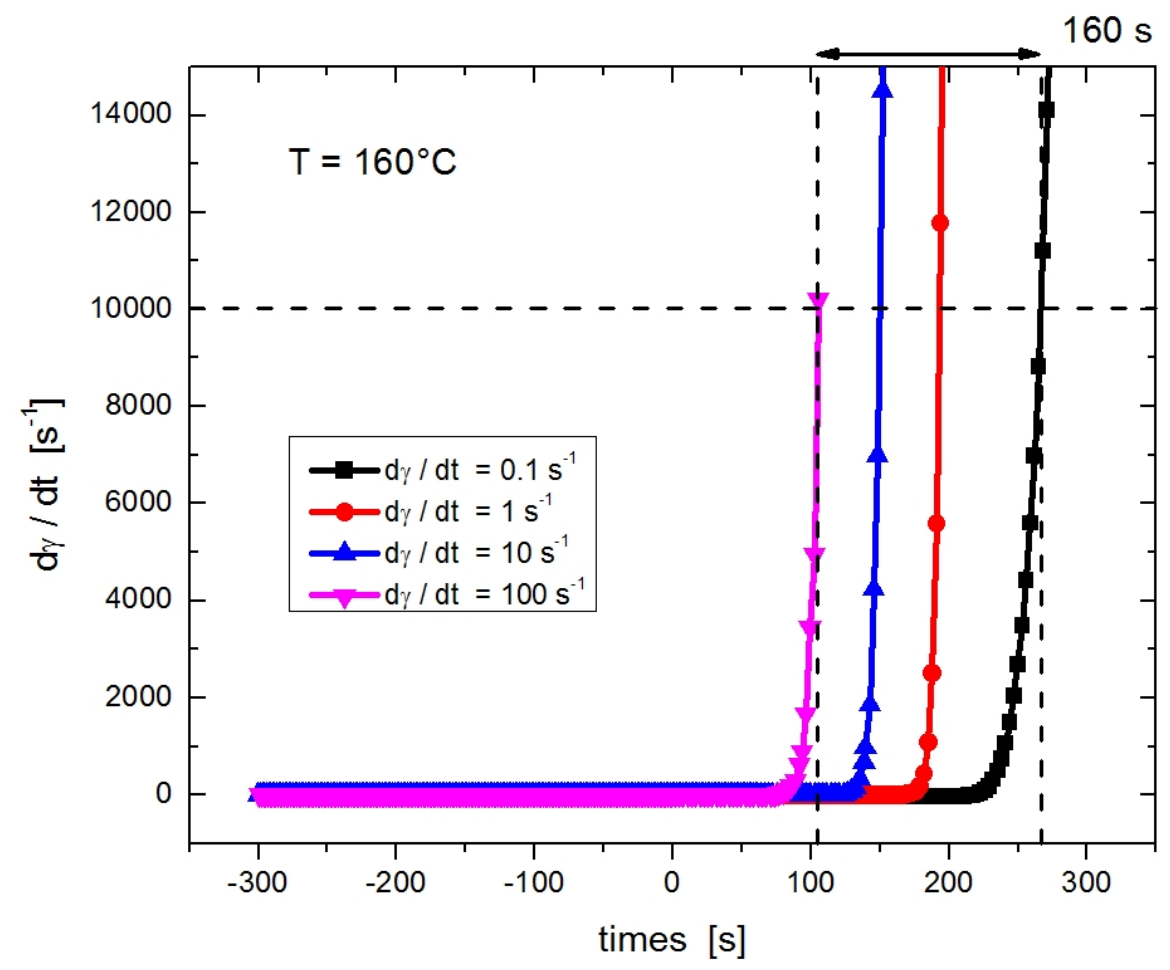

Figure 5. Viscosity evolution at different shear rates as function of time at a constant polymerization temperature $\mathrm{T}_{\text {polym }}=160^{\circ} \mathrm{C}$. 


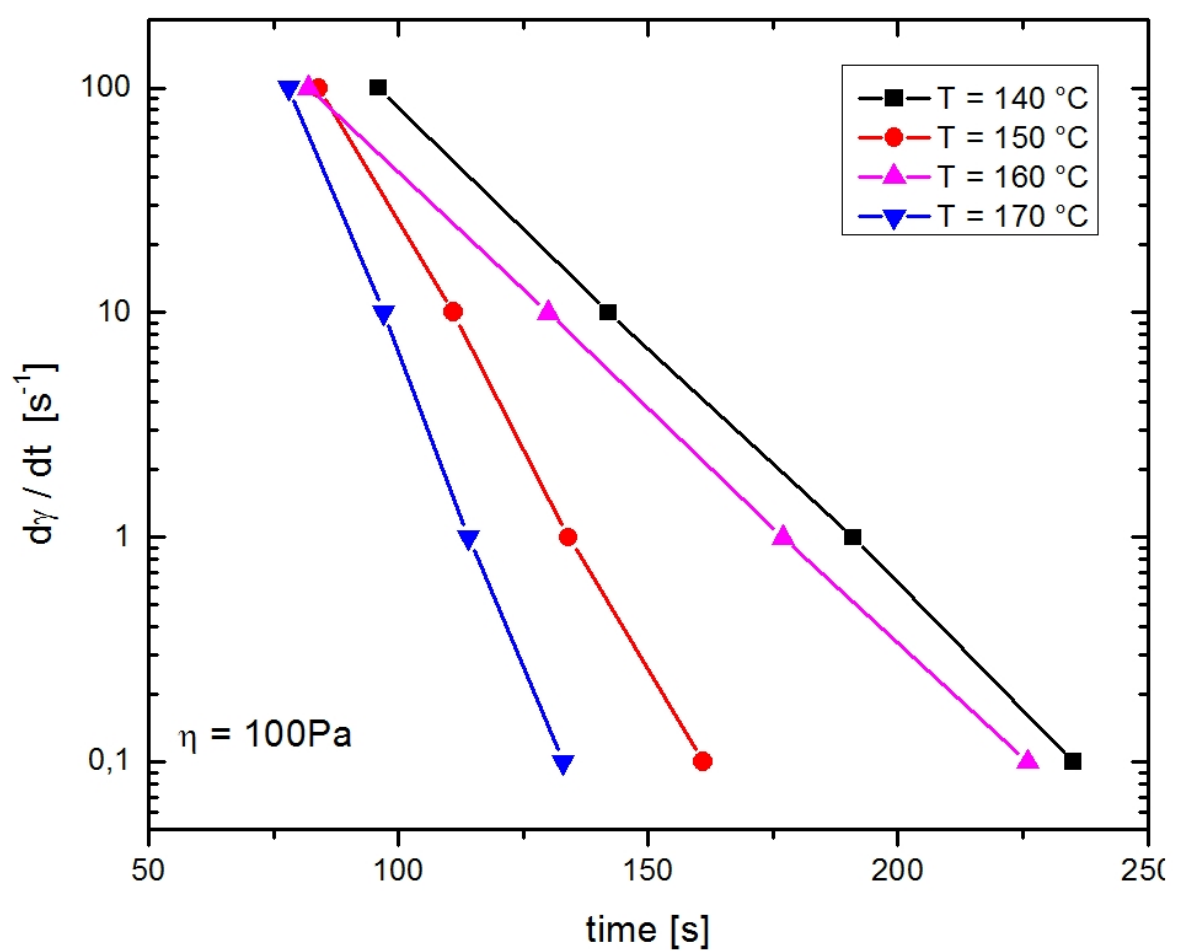

Figure 6. Shear rate $\dot{\gamma}$ chart representing a function of time to reach an arbitrary viscosity $\eta=100 \mathrm{~Pa}$.s for four predefined polymerization temperatures.

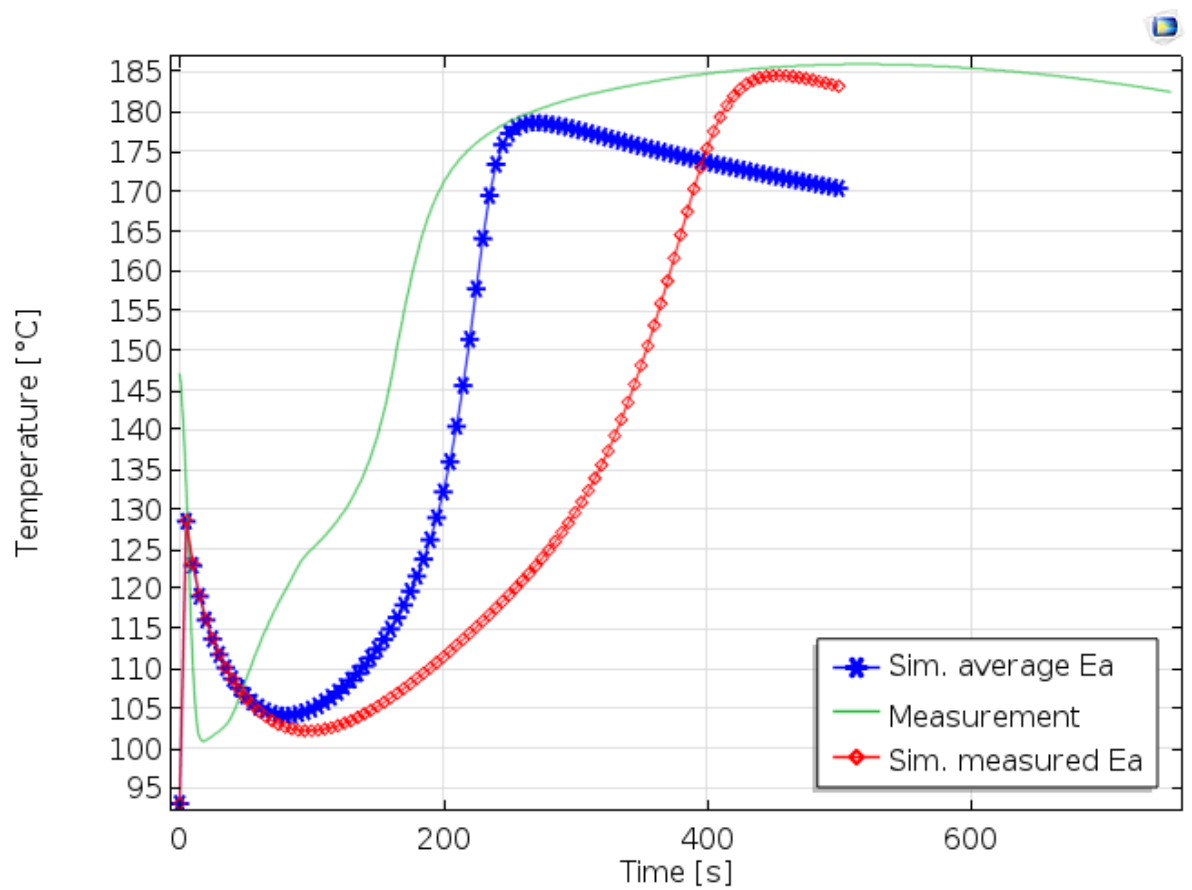

Figure 7. Comparison of the measured values and the numerical prediction based on the own measured activation energies and average values from [15] 

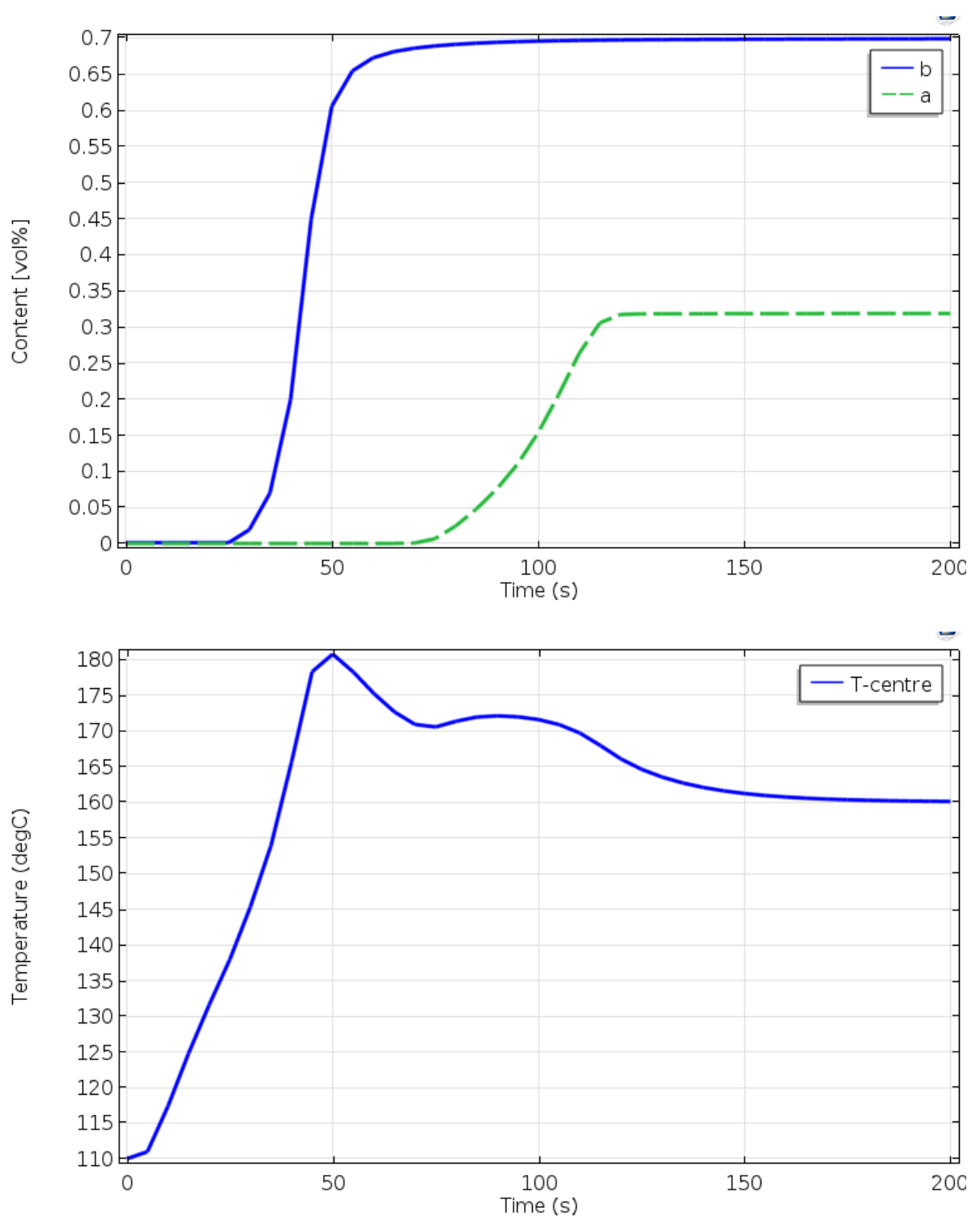

Figure 8. Numerical prediction of the phase contents [top] and of the temperature evaluation [bottom] inside the instrumented mold [15] 


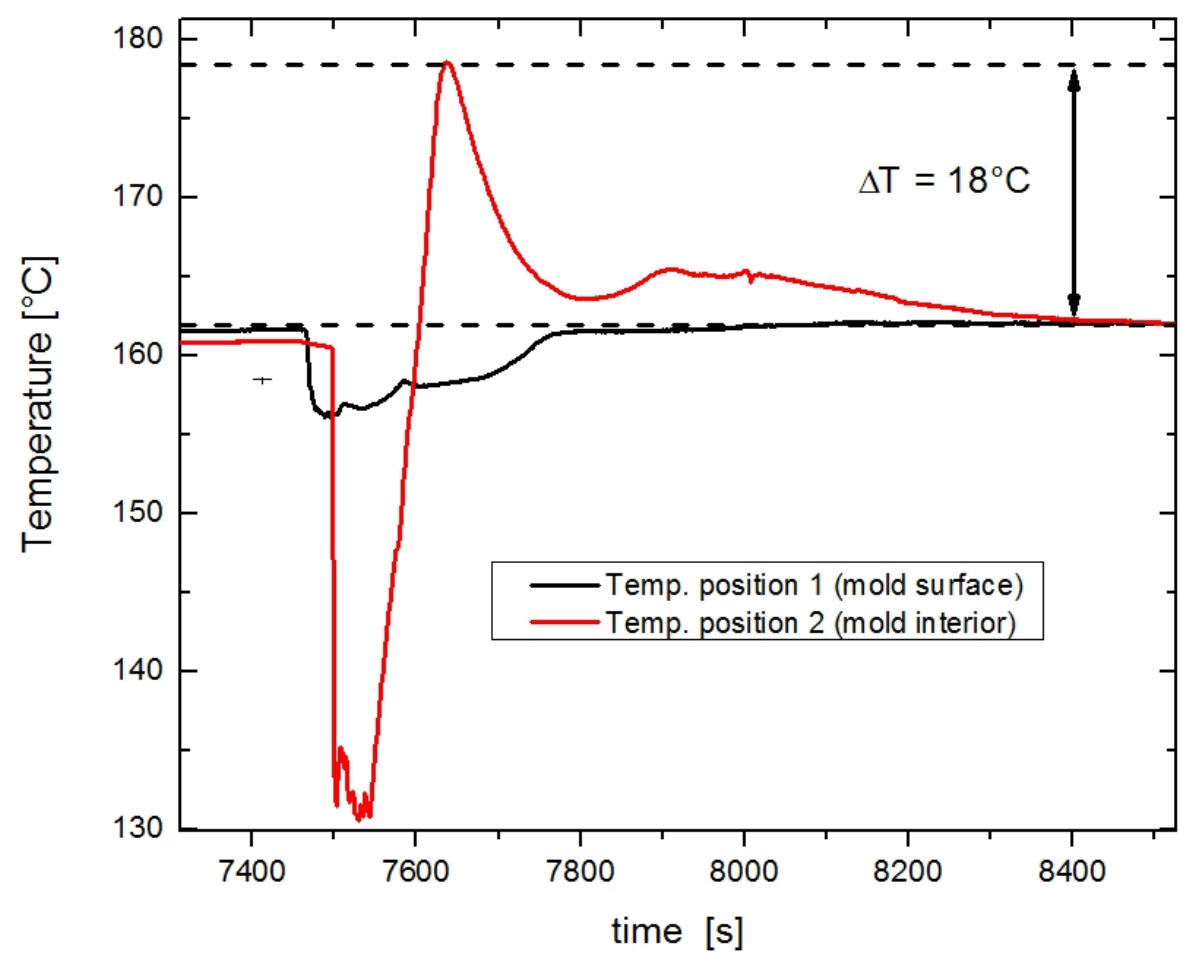

Figure 9. Surface temperature evolution at the surface (black line) and in the bulk (red line) during an injection into a preheated mold at $\mathrm{T}=160^{\circ} \mathrm{C}$.

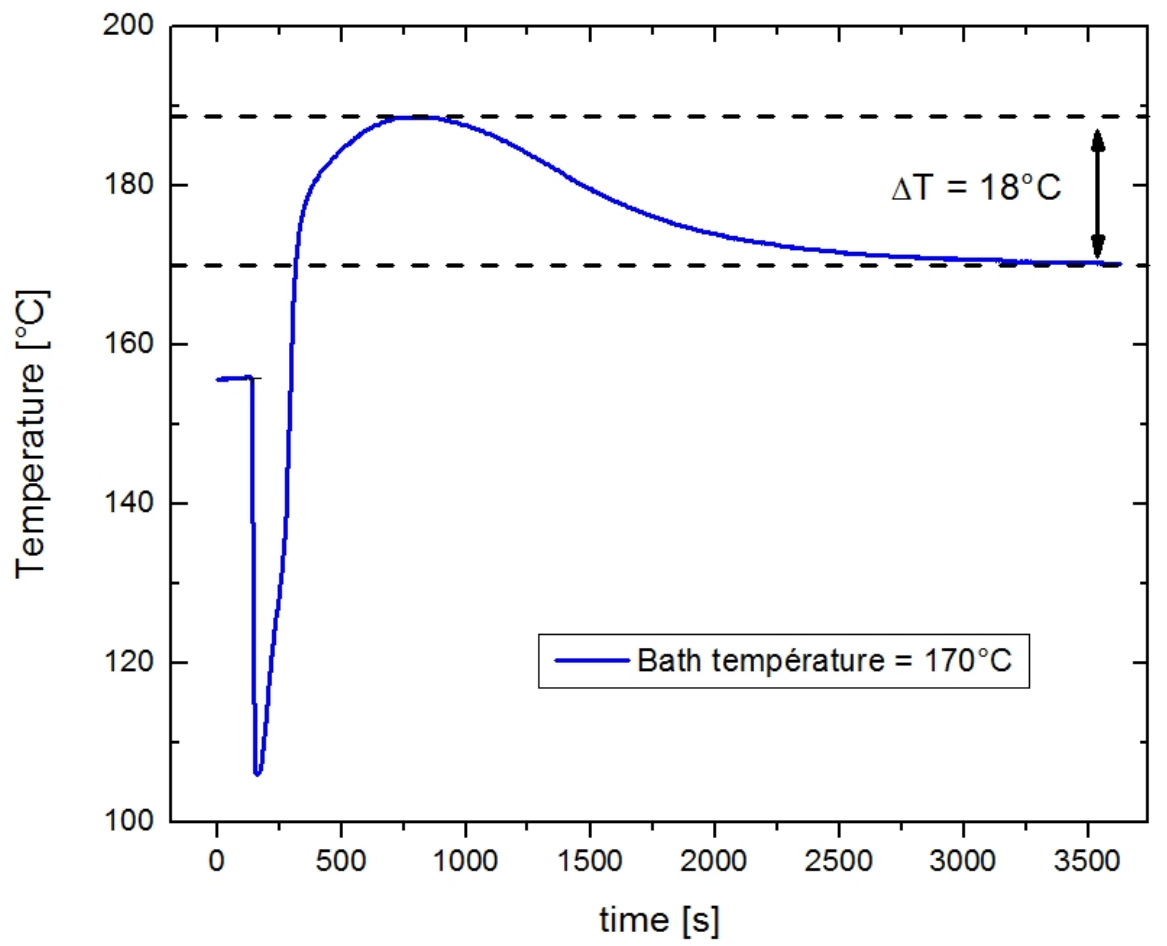

Figure 10. [top] Recorded curve in the thermal reactor for a bath temperature of $\mathrm{T}=170^{\circ} \mathrm{C}$. 


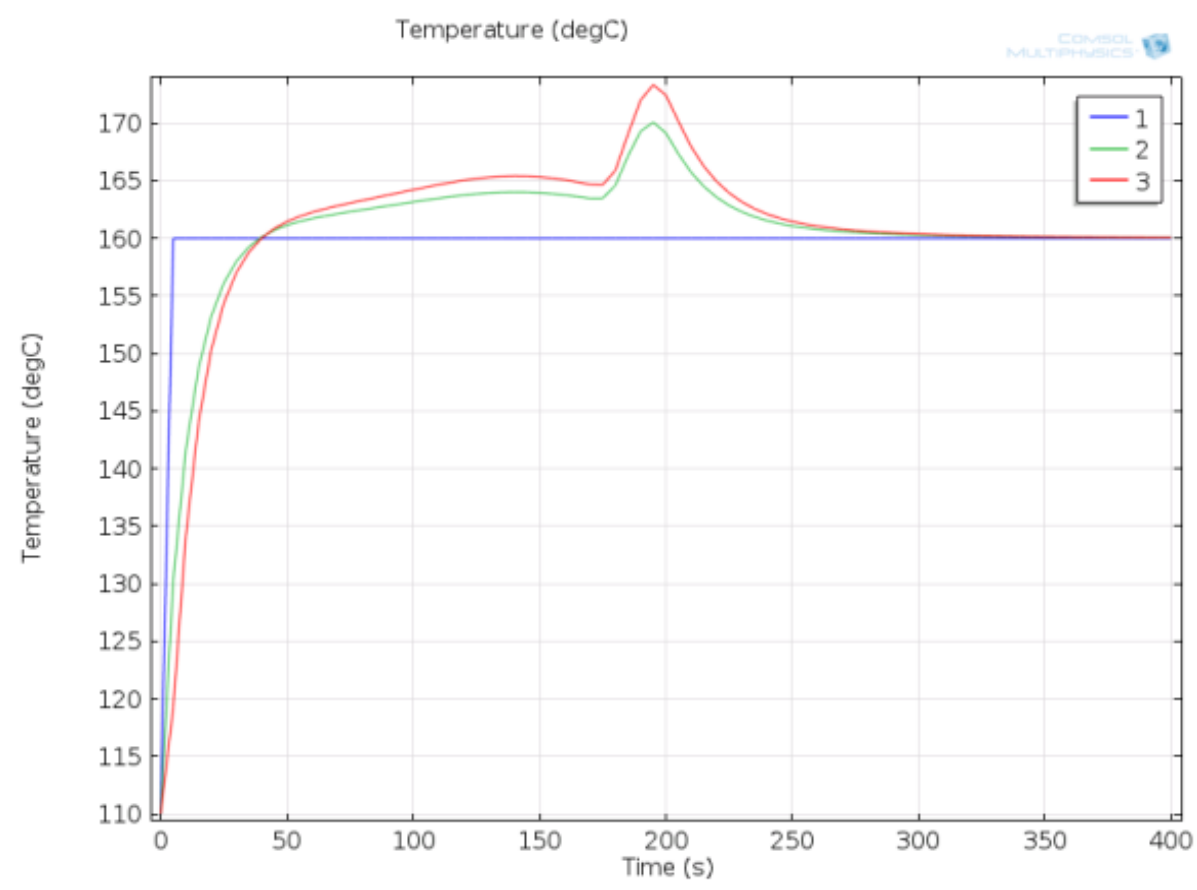

Figure 11. Simulation of the temperature built up in the same experimental conditions.

The time to reach a viscosity of $\eta=100 \mathrm{~Pa}$.s is comprised between $t=75 \mathrm{~s}$ and $t=250 \mathrm{~s}$. The knowledge of this time interval is important to define a time lag during which the viscosity of reactive mixture is industrially feasible. The lower the polymerization temperature and shear rate will be the longer is the polymerization time. Conversely, the higher the polymerization temperature and shear rate will be, the shorter the cure time will be. These statements are however of limited influence for a moderate shear rate of $\dot{\gamma}=100 \mathrm{~s}^{-1}$ as the time difference is only of $\Delta t=16 \mathrm{~s}$ between two polymerization temperatures $\mathrm{T}=140^{\circ} \mathrm{C}$ and $\mathrm{T}=170^{\circ} \mathrm{C}$. Nevertheless, at a shear rate $\dot{\gamma}=0.1 \mathrm{~s}^{-1}$, this influence is more clearly observable since the time difference between the polymerization temperature $\mathrm{T}=140^{\circ} \mathrm{C}$ and $\mathrm{T}=170^{\circ} \mathrm{C}$ is of $\Delta t=100 \mathrm{~s}$. Similarly, for this viscosity of reference, the polymerization times are longer at $\mathrm{T}=160^{\circ} \mathrm{C}$ than at $\mathrm{T}=150^{\circ} \mathrm{C}$, over almost the entire shear rate range. This can be explained by the fact that the polymerization temperature $\left(\mathrm{T}=150^{\circ} \mathrm{C}\right)$ is critical to the system. Indeed, it is well known from the literature [14] that this is temperature range of maximum of the crystallization.

Both kinetic scales (polymerization and crystallization) in this temperature range are temperature-sensitive, but also sensitive to the applied shear rate during polymerization. From a molecular point of view, the greater the shear rate, the higher the probability of contacting the activator molecules, initiator and monomer will be and will thus facilitate the polymerization system. It can be concluded that the implementation of a quicker movement of the measuring cells has an effect accelerator/inhibitor on the time of polymerization and those regardless of the polymerization temperature. This suggests that the shear rate is important in the mixing of the constituents. Taking into account the results, the shear rate of the resin during the implementation will be privileged to trapping of the active ingredients in the delay strategy polymerization.

\subsection{Macroscopic Characterizations}

To achieve the macroscopic characterization of the plates made in the instrumented mold, six characteristics regions were chosen for analysis: along the plate axis and along on edge. Studies of the degree of conversion and of crystallinity were conducted at each area to map and have an accurate physicochemical picture of each plate.

It was found that the degree of conversion vary between $\beta=63.9 \%$ and $\beta=84.1 \%$. There is a dependence of the conversion rate between the axis and the edge of the plate. The conversion rates at edge plate are higher and less dispersed than in the center. It was found that the degree of crystallinity vary between $X_{\mathrm{c}}=23.5 \%$ and $X_{\mathrm{c}}=32.5 \%$. It exists a much larger disparity in the degree of conversion than in the degree of crystallinity.

The differences in polymerization rate are likely due to the heterogeneity of the polymerization temperature during injection. However, this disparity in the rate of polymerization may be reduced with the approach developed for controlling the reaction, while the differences in the degree of crystallinity can be improved by thermal post-polymerization treatments.

\subsection{Simulation}

To validate the model and the measured values the thermal reactor for a bath temperature of $\mathrm{T}=160^{\circ} \mathrm{C}$ is simulated. The final degree of polymerization is given with $\beta=70 \%$ and the 
degree of crystallization with $X_{c}=33 \%$. As the prediction of the temperature is not very well using the own measured activation energy, average values of Kamal-Sourour parameters for the same polymer system are taken from [15].

Figure 7 shows both simulation results. The prediction based on the average literature values for $\mathrm{E}_{a}$ is much closer to the measured values. There is still a difference in the prediction of the temperature before the reaction.

Being closer to the real part production, the temperature evaluation in the instrumented mold is simulated. Validating only the thermal heat transfer in the thickness, a 1D model representing the $4 \mathrm{~mm}$ thick mold is simulated. The initial temperature of the mold is $\mathrm{T}=160^{\circ} \mathrm{C}$ and the one of the polymer $\mathrm{T}=110^{\circ} \mathrm{C}$. The degree of polymerization is $\beta=70 \%$ and the one of the crystallization is $X_{c}=33 \%$. The numerical prediction of the maximal temperature is very close to the experimentally measured $\mathrm{T}=178^{\circ} \mathrm{C}$ (see Fig. 1). The first peak corresponding to the polymerization is followed by a second one representing the heat of the crystallization. The degree of polymerization $\beta$ increases as soon as T $>140^{\circ} \mathrm{C}$. At $t=45 \mathrm{~s}$ the crystallization starts and $\beta$ decreases by the amount of $\alpha$. The crystallization stops when $\alpha_{e q}$ is reached (see Fig.8).

\subsection{Validation of Theoretical Models by Controlled Tests in an Instrumented Mold}

Following the completion of the instrumented mold, controlled tests have been carried out. It was thus possible to correlate injection reality with injection predictions of the simulation. Two recorded curves in the instrumented mold during an injection are shown in figure 9 .

The reactive resin arrival on the temperature sensor in position 2 locally drops down the temperature. This sensor shows that the reaction liquid flows with a temperature $\mathrm{T}=130^{\circ} \mathrm{C}$ and not at $\mathrm{T}=110^{\circ} \mathrm{C}$. The front of the mixture increases with increasing temperature in the mold. This data will have a direct impact on the performance and quality of the material (conversion rate and crystallinity). The red curve also indicates, by the sudden rise of the temperature, the polymerization of the material. It takes place $t=50 \mathrm{~s}$ after the detection of the flow front. Here, the polymerization is exothermic in mold center and reach a temperature $\mathrm{T}=178^{\circ} \mathrm{C}$. On the surface, the maximum recorded temperature is $\mathrm{T}=165^{\circ} \mathrm{C}$.

This exothermic effect during polymerization was also recorded in the thermal reactor (see Fig.10) and highlighted by the simulation with the following parameters (see Fig.11):

1) Degree of crystallinity $X_{c}=33 \%$

2) The rate of polymerization $\beta=80 \%$ (measured rate in the plates).

In figure 10, the curve was recorded at the center of a thermal reactor placed into a bath temperature at $\mathrm{T}=170^{\circ} \mathrm{C}$. It is noted that there is a $\Delta \mathrm{T}=18^{\circ} \mathrm{C}$ between the maximum of the exothermic and the temperature of the bath. This temperature difference is similar to that found in the instrumented mold.
In figure 11, curve noted 3 correspond to the simulation of changes in the temperature at the center of the mold during the polymerization. As in reality, (thermal reactor and instrumented mold) curve 3 shows a temperature built up which is equal to $\Delta \mathrm{T}=18^{\circ} \mathrm{C}$ between the maximum of the exothermic and the temperature of the bath.

Thus, through these areas, there is a consistency between the reality of the injection and the simulation of the polymerization in the evolution of the measured temperatures.

\section{Conclusions}

Operating at polymerization temperatures below the melting temperature of the considered thermoplastic AP-Nylon ${ }^{\circledR}$, there exists a strong competition between the polymerization kinetic and crystallization kinetic during injection into a thick heterogeneous and poorly conductive medium.

The reactive resin permanently evolves throughout the injection. The presence of spatial heterogeneity will be symbolized by the existence of non-polymerized parts, polymerized zones, crystalline domains and fiber reinforcement in case of composites. These spatial heterogeneity problems inevitably will induce diffusion of the reactive resin and problems with the reinforcement's impregnation during the production of thick parts.

Moreover, low thermal conductivity of polymers and the strong exothermal effect generated by the polymerization will cause the presence of strong temperature gradients during the injection and therefore, the existence of a thermal heterogeneity in the entire sample. As the viscosity evolution is directly linked to the chemical reaction, these spatial and thermal heterogeneities create inevitably a non-homogenous piece.

In order to develop and produce the most homogeneous pieces in conversion rate and degree of crystallinity, it is necessary to develop a new strategy of the T-RTM injection based on the control of the reactive mixture. For this, many scientific uncertainties have to be lifted and go deeper in the knowledge and the understanding of chemical, physico-chemical and rheological phenomena of this reactive mixture occurring during the polymerization reaction.

In this work, a better understanding of the physico-chemical and rheological phenomena ruling the anionic polymerization reaction of a lactam ( $\varepsilon$-caprolactam) to synthesize a thermoplastic composite (PA6) was achieved. The crystallization and polymerization kinetics study has demonstrated the existence of a new characteristic reaction parameter, other than the polymerization temperature, the applied shear rate to the sample. It has been shown that it is possible to homogenize and regulate the chemical reaction progress during the injection process in order to uniformize the temperature within thick pieces.

To support these experimental results, a computer 
simulation of the injection process has been established. This simulation takes into account predefined physical and chemical models related to existing flow phenomena occurring during the T-RTM injection. This computer model opens new ways to characterize and model thermoplastic injections. Nevertheless, to fully control the process, additional analysis of temperature during T-RTM in the molds need to be done.

Up to now, injection methods are clearly established for thermosets but are far from being adapted to thermoplastics. However, the improvements of the temperature and shear rate in T-RTM reactive injection processing are real technological and scientific benefits. All these results and computer-assisted simulations will have been define the optimized parameters of reactive injection and, only then, this method could be transferred. Hence, they will adjust the various parameters to current technical and industrial issues.

\section{REFERENCES}

[1] S. Pillay, Liquid Molding of Carbon Fabric-reinforced Nylon Matrix Composite Laminates, Journal of Thermoplastic Composite Materials, 18, 2005.

[2] W. D. Brouwer, E. C. F. C. van Herpt, and M. Labordus, Vacuum injection moulding for large structural applications, Composites Part A: Applied Science and Manufacturing, 34, 2003.

[3] Y. Gong and G. Yang, All-polyamide composites prepared by resin transfer molding, J Mater Sci, 45, 2010.

[4] K. van Rijswijk and H. E. N. Bersee, Reactive processing of textile fiber-reinforced thermoplastic composites - An overview, Composites Part A: Applied Science and Manufacturing, 38, 2007.

[5] J. Verrey, M. D. Wakeman, V. Michaud, and J. A. E. Manson, Manufacturing cost comparison of thermoplastic and thermoset RTM for an automotive floor pan, Composites Part A: Applied Science and Manufacturing, 37, 2006.
[6] J.A.E. Manson, New demands on manufacturing of composite materials, Conference: Materials week '94, Rosemont, IL (United States), 3-7 Oct 1994, ISBN 0-87339-247-7.

[7] S.G. Advani, M.V. Bruschke, R.S. Parnas, Resin transfer molding flow phenomena in polymeric composites, Flow and Rheology in Polymer Composite Manufacturing, Elsevier, 1994.

[8] R. S. Davé, R. L. Kruse, L. R. Stebbins, and K. Udipi, Polyamides from lactams via anionic ring-opening polymerization: 2. Kinetics, Polymer, 38, 1997.

[9] H. C. Y. Cartledge and C. A. Baillie, Studies of microstructural and mechanical properties of nylon/glass composite Part I, J Mater Sci, 34, 1999.

[10] K. H. Lee and S. C. Kim, Reaction-induced crystallization kinetics during the anionic polymerization of $\varepsilon$-caprolactam, Polym. Eng. Sci., 28, 1988.

[11] J. J. E. Teuwen, A. A. van Geenen, and H. E. N. Bersee, Novel Reaction Kinetic Model for Anionic Polyamide-6, Macromol. Mater. Eng., 298, 2012.

[12] A. Rigo, G. Fabbri, and G. Talamini, Kinetic study of anionic polymerization of 6-caprolactam by differential calorimetry, J. Polym. Sci. B Polym. Lett. Ed., 13, 1975.

[13] A. Y. Malkin, S. L. Ivanova, V. G. Frolov, A. N. Ivanova, and Z. S. Andrianova, Kinetics of anionic polymerization of lactams. Solution of non-isothermal kinetic problems by the inverse method, Polymer, 23, 1982.

[14] R. S. Davé, R. L. Kruse, K. Udipi, and D. E. Williams, Polyamides from lactams via anionic ring-opening polymerization: 3. Rheology, Polymer, 38, 1997.

[15] K. van Rijswijk, H. E. N. Bersee, A. Beukers, S. J. Picken, and A. A. van Geenen, Optimisation of anionic polyamide- 6 for vacuum infusion of thermoplastic composites: Influence of polymerisation temperature on matrix properties, Polymer Testing, 25, 2006.

[16] L. Ricco, S. Russo, G. Orefice, and F. Riva, Anionic

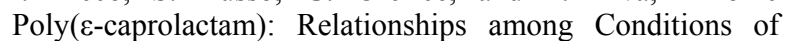
Synthesis, Chain Regularity, Reticular Order, and Polymorphism, Macromolecules, 32, 1999. 